\title{
Mechanism Design via Correlation Gap
}

\author{
Qiqi Yan* \\ qiqiyan@cs.stanford.edu \\ Department of Computer Science \\ Stanford University
}

October 22, 2018

\begin{abstract}
For revenue and welfare maximization in singledimensional Bayesian settings, Chawla et al. (STOC10) recently showed that sequential posted-price mechanisms (SPMs), though simple in form, can perform surprisingly well compared to the optimal mechanisms. In this paper, we give a theoretical explanation of this fact, based on a connection to the notion of correlation gap.

Loosely speaking, for auction environments with matroid constraints, we can relate the performance of a mechanism to the expectation of a monotone submodular function over a random set. This random set corresponds to the winner set for the optimal mechanism, which is highly correlated, and corresponds to certain demand set for SPMs, which is independent. The notion of correlation gap of Agrawal et al. (SODA10) quantifies how much we "lose" in the expectation of the function by ignoring correlation in the random set, and hence bounds our loss in using certain SPM instead of the optimal mechanism. Furthermore, the correlation gap of a monotone and submodular function is known to be small, and it follows that certain SPM can approximate the optimal mechanism by a good constant factor.

Exploiting this connection, we give tight analysis of a greedy-based SPM of Chawla et al. for several environments. In particular, we show that it gives an $e /(e-1)$-approximation for matroid environments, gives asymptotically a $1 /(1-1 / \sqrt{2 \pi k})$-approximation for the important sub-case of $k$-unit auctions, and gives a $(p+1)$-approximation for environments with $p$ independent set system constraints.
\end{abstract}

\section{Introduction}

In mechanism design, or even more broadly in algorithm design as well, there is an inherent conflict be-

\footnotetext{
*This research was supported by a Stanford Graduate Fellowship. Part of this research was done while the author was at Yahoo! Research, Santa Clara.
}

tween optimality and simplicity. Mechanisms like Myerson's mechanism Mye81 or the VCG mechanism Vic61, Cla71, Gro73 have optimal revenue or welfare guarantees, but often suffer from having complicated formats or severe computational overhead. For example, even in single-item auctions, the need for the agents to commit to the auction process itself can be a significant burden AM06, Hol08, and in combinatorial auctions, determining the allocation and payments of the VCG mechanism is a computationally hard problem [NR00]. Therefore, not surprisingly, simple mechanisms are very often favored in practice [Hol08]. Consider sequential posted-price mechanisms, in which the seller makes take-it-or-leave-it price offers to agents one by one. Such mechanisms are easy to run for the sellers, leave little room for agents' strategic behavior, and keep the information elicitation from the agents at a minimum level. Of course, simplicity comes at a cost, as such simple mechanisms are in general not optimal. Therefore, it is an interesting question to quantify how much we are paying for keeping it simple.

Following a recent trend [HR09, DRY10, CHMS10, we focus on quantifying the performance (revenue or welfare) of simple mechanisms relative to that of the optimal mechanism. In particular, we focus on revenue and welfare maximization in single-dimensional Bayesian mechanism design, and we are interested in comparing the performance of Sequential Posted-price Mechanisms (SPMs) to that of the optimal mechanism, which is Myerson's mechanism for revenue, and the VCG mechanism for welfare. In a recent work of Chawla et al. CHMS10, it was shown for several contexts that the performance of a SPM (which we call greedy-SPM) approximates that of the optimal mechanism by a small constant factor, where the factor is 2 for matroid environments (which generalize $k$-unit auctions, certain matching markets etc.), and $e /(e-1)$ for $k$-unit auctions. This is surprising, as SPMs can only offer prices to agents in a very restricted way, while the optimal 
mechanism can choose a price for each agent based on full information about all other agents. What is the underlying reason for SPMs' good performance? Our main goal of this paper is to give a theoretical explanation for this curious fact, based on a connection to the notion of correlation gap.

Reducing Mechanism Design to Correlation Gap The notion of correlation gap was first formalized in Agrawal et al. ADSY10. Let $f(S)$ be a function that maps a subset $S$ of a finite ground set $N$ to a nonnegative real number. For $\mathcal{D}$ a distribution over $2^{N}$ with marginal probabilities $q_{i}=\operatorname{Pr}_{S \sim \mathcal{D}}[i \in S]$, let $\mathcal{I}_{\mathcal{D}}$ be the independent distribution where each $i \in N$ is included in the set with the same marginal probability $q_{i}$, but independently. The correlation gap of $f$ is defined as the supremum of $\frac{E_{S \sim \mathcal{D}}[f(S)]}{E_{S \sim \mathcal{I}(\mathcal{D})}[f(S)]}$ over all distribution $\mathcal{D}$, which in some sense bounds our "loss" in expected value of the function by ignoring correlation.

Loosely speaking, the approximation ratio of SPMs w.r.t. the optimal mechanism is related to correlation gap in the following way. The performance of a mechanism can often be related to the expectation of certain function $f$ over a random set of agents. For the optimal mechanism, this random set corresponds to the set of winners, while for an SPM, this random set corresponds to the demand set, which is the set of agents whose values beat the prices set for them in the SPM. Notice that the winner set is highly-dependent, while the demand set is independent. By setting prices for agents in an SPM carefully such that these two random sets have the same marginal probabilities, we can apply the correlation gap of $f$ to get a bound on the approximation ratio of the SPM w.r.t. the optimal mechanism.

Reduction for $k$-Unit Auctions To illuminate the idea, suppose we sell $k$ items to a set of $n$ agents $N=$ $\{1, \ldots, n\}$ with valuations drawn i.i.d. from a normal distribution $F$, and our goal is to maximize expected revenue. Define set function $f$ as $f(S)=\min (|S|, k)$ for $S \subseteq N$. Let $q$ be the probability that Myerson's optimal mechanism sells to a particular agent (which is the same for every agent by symmetry). It can be shown that the optimal way to sell to an agent with success probability $q$ in an incentive compatible manner is to offer the deterministic price $p=F^{-1}(1-q)$. Therefore if we pretend that an agent pays $p$ whenever she wins in the optimal mechanism, the total calculated revenue is only higher. In other words, the revenue of Myerson's mechanism is upper-bounded by $E_{W}[f(W)] \cdot p$, where $W$ is the set of winners. On the other hand, let an SPM make take-it-or-leave-it offers at price $p$ to every agent sequentially. Define demand set $D$ as the set of agents whose values are at least $p$. Since at most $k$ agents can be served, the revenue of the SPM is equal to $E_{D}[f(D)] \cdot p$. Note that $W$ and $D$ have the same marginal probability $q$ for every $i$, and $D$ follows an independent distribution. Therefore if we can show that the correlation gap of $f$ is at most $\beta$, then $E_{D}[f(D)] \geq(1 / \beta) \cdot E_{W}[f(W)]$, and it follows that the revenue of SPM is a $\beta$-approximation to that of Myerson's mechanism.

Submodularity The set function $f$ that arises in our context is the weighted rank function of the set system that encodes the feasibility constraints of the environment. For settings where constraints are modeled by matroids, the weighted rank functions are well-known to be monotone and submodular. This fact enables us to invoke a deep result from the literature on submodular functions Von07, ADSY10, which says that the correlation gap of a monotone and submodular function is at most $e /(e-1)$. It follows that for matriod environments, SPMs can approximate the optimal mechanism by a factor of $e /(e-1)$. This result would be otherwise difficult to achieve without making use of our explicit connection to correlation gap and submodularity.

Recognizing submodularity is also helpful in other ways. In the analysis for $k$-unit auctions, we exploit the cross-convexity of the multi-linear extension of submodular functions to get a tight bound on the correlation gap of the corresponding weighted rank function.

Applying the Reduction The reduction to correlation gap gives us a structured way of analyzing greedy-SPM. It abstracts away all the mechanism design aspects of the problem, such that we can focus on the purely mathematical question of quantifying correlation gaps of weighted rank functions. Based on this approach, we give tight analysis for greedy-SPM in several contexts. In the following, approximation guarantees are for an objective that can be revenue or welfare or certain combination of both, and are for the version of greedy-SPM that is tailored to the objective.

For matroid environments, as mentioned above, we show that greedy-SPM is a $e /(e-1)$-approximation to the optimal mechanism, an improvement over the previous 2-approximation. For the important sub-case of $k$-unit auctions, we show that greedy-SPM has approximation ratio $1 /\left(1-\frac{k^{k}}{e^{k} k !}\right) .\left(\approx 1 /\left(1-\frac{1}{\sqrt{2 \pi k}}\right)\right.$ by Stirling's formula) This implies that the performance of SPMs can approach that of the optimal mechanism as the supply increases. In particular here we do not assume that agents' valuation distributions are identical. Towards settings more general than matroid environments, we study $p$-independent environments, where feasibility constraints are modeled by $p$-independent set systems, a generalization of intersection of $p$ matroids. In such settings, we show that correlation gap is at most $p+1$, 
which also translates into a $(p+1)$-approximation for greedy-SPM. This generalizes the result on intersection of $p$ matroids in [CHMS10].

1.1 Related Work For the maximization of revenue and welfare, Myerson's mechanism Mye81, BR89 and the VCG mechanism [Vic61, Cla71, Gro73] are optimal, respectively. Recent work in the CS literature has focused on designing simple mechanisms that are approximately-optimal, while being more detail-free or robust. Myerson's mechanism in general involves the calculation of (ironed) virtual valuations using full distribution information. Hartline and Roughgarden HR09 showed that a simpler mechanism, namely the VCG mechanism with monopoly reserves is approximately optimal by a constant factor for many natural settings, and Dhangwatnotai et al. [DRY10] further removed the need of knowing monopoly reserves in advance via a sampling-based approach. In another direction, Sundararajan and Yan SY10 studied mechanisms that are approximately optimal for utility-maximizing risk-averse sellers, even without prior knowledge about their concave utility functions.

Sequential posted-price mechanisms have also been a recent focus of study due to their simplicity and various appealing properties. Blumrosen and Holenstein [BH08 first compared SPMs to Myerson's mechanism for single-item auctions by an asymptotic analysis. Chawla et al. CHMS10 studied SPMs in various auction contexts, proving that SPMs perform very well compared to Myerson's mechanism, which motivated our work. They also used SPMs as a building block to construct approximately-optimal mechanisms in multi-dimensional settings. Independent of our work, Chakraborty et al. [CEDG ${ }^{+} 10$ proved almost the same approximation guarantee for $k$-unit auctions. They also studied SPMs that adaptively choose prices and the ordering of agents. Babaioff et al. [BBDS11] studied adaptive SPMs in settings where agents' valuations are drawn i.i.d. from an unknown distribution. In other aspects, Sundararajan and Yan SY10] studied the performance of SPMs when the sellers are risk-averse, and aim to maximize expected utility.

There is a vast literature on the study of submodular functions (see references in [Von07). The correlation gap of monotone submodular functions was first bounded in [CCPV07, and it is also tightly related to the submodular welfare maximization problem Von08. In the context of auctions, Dughmi et al. DRS09 showed that in matroids environments, the revenue of Myerson's mechanism is submodular in the set of agents that we actually run the mechanism over.

\section{Preliminaries}

Auction Environments In our setting, the seller sells services (or goods) to a set of $n$ unit-demand agents $N=\{1, \ldots, n\}$. Each agent $i$ has a private valuation $v_{i}$ for winning the service, and 0 otherwise, where each $v_{i}$ is drawn independently from a known distribution $F_{i}$. For simplicity we assume that every distribution is over a finite support $[0, L]$ for some large $L$, and has a positive smooth density function. It is only feasible for the seller to service certain subsets of the agents simultaneously, and we let $\emptyset \in \mathcal{I} \subseteq 2^{N}$ represent all the feasible subsets. We assume that the environment is always downwardclosed, in the sense that the subset of a feasible set is also feasible. Auction environments are classified by the set systems $(N, \mathcal{I})$. In particular we study $k$-unit auctions, where a set $S$ is in $\mathcal{I}$ if and only if $|S| \leq k$, matroid environments, where $(N, \mathcal{I})$ forms a matroid, and $p$-independent environments, where $(N, \mathcal{I})$ form a $p$-independent set system. We will define the latter two environments later.

Mechanisms A (deterministic) mechanism uses an allocation rule $\mathbf{x}:[0, \infty)^{n} \rightarrow\{0,1\}^{n}$ to choose the (characteristic vector of) winning set of agents based on the reported valuations $\mathbf{v} \in[0, \infty)^{n}$ of the agents, and uses a payment rule $\mathbf{p}:[0, \infty)^{n} \rightarrow[0, \infty)^{n}$ to charge payments from the agents. A randomized mechanism is a distribution over deterministic mechanisms. For ease of presentation, we study mechanisms that are incentive compatible (a.k.a., truthful) and individual rational, both in the ex post sense, although our results still hold if we allow mechanisms to be Bayesian incentive compatible. An equivalent way of defining ex post incentive constraints is that for each agent $i$, if we fix the valuations $\mathbf{v}_{-i}$ of the other agents, agent $i$ faces a takeit-or-leave-it offer at a price $p_{i}\left(\mathbf{v}_{-i}\right)$ that is independent of agent $i$ 's own value $v_{i}$.

Given an ordering of agents and a price $p_{i}$ for each agent $i$, a Sequential Posted-price Mechanism (SPM) first initializes the allocated set $A$ to be $\emptyset$, and for all agents $i$ in the given order, do the following: if serving $i$ is feasible, i.e., $A+i \in \mathcal{I}$, offer to serve agent $i$ at the pre-determined price $p_{i}$, and add $i$ to $A$ if agent $i$ accepts. A randomized SPM is then a distribution over deterministic SPMs.

Weighted Rank FunctionsFor a set system $(N, \mathcal{I})$ with nonnegative weights $\left(w_{i}\right)_{i \in N}$ on the elements, we define the weighted rank function $w^{*}(S)$ as the maximum of $\sum_{i \in T} w_{i}$ over all $T \subseteq S$ with $T \in \mathcal{I}$. The (unweighted) rank functions are defined with weights set to 1 .

Greedy Given a set system $(N, \mathcal{I})$ with nonnegative weights $\left(w_{i}\right)_{i \in N}$, and a subset $S$ of $N$, the greedy algorithm starts with an empty solution set $A$, and for 
each agent $i$ in $S$ in decreasing order of $w_{i}$, adds $i$ into the solution set $A$ whenever $A \cup\{i\}$ is in $\mathcal{I}$. Finally it outputs $A$. We let greedy $(S)$ denote the final output of greedy algorithm.

Matroids A set system $(N, \mathcal{I})$ is a matroid system (see e.g. Oxl92 if (1) $S \in \mathcal{I}$ whenever $S \subseteq T \in \mathcal{I}$, and (2) if $S, T \in \mathcal{I}$ and $|S|>|T|$, then for some $e \in S \backslash T$, $T \cup\{e\} \in \mathcal{I}$. We will make use of the following two well-known properties about matroids: (1) If we run greedy on a subset $S$ of the matroid, then the weight of its output set equals to the weighted rank of the set $S$, i.e., $\sum_{i \in \operatorname{greedy}(S)} w_{i}=w^{*}(S)$. (2) The weighted rank function of a matroid is monotone and submodular.

Correlation Gap and Submodularity Given a set function $f: 2^{N} \rightarrow[0, \infty)$ over a finite set $N$, let $\mathcal{D}$ be a distribution over $2^{N}$ with marginal probabilities $\mathbf{q}=\left(q_{i}\right)_{i \in N}$. Let $S \sim \mathcal{I}(\mathcal{D})$ denote that each $i \in N$ is included in $S$ with probability $q_{i}$ independently. Then the correlation gap ADSY10 11 of $f$ is $\sup _{\mathcal{D}} \frac{E_{S \sim \mathcal{D}}[f(S)]}{E_{S \sim \mathcal{I}(\mathcal{D})}[f(S)]}$. (we let $\frac{0}{0}=1$ here)

A set function $f: 2^{N} \rightarrow[0, \infty)$ is monotone if $f(S) \leq f(T)$ whenever $S \subseteq T$, and is submodular if $f(S)+f(T) \geq f(S \cup T)+f(S \cap T)$ for all $S, T$.

THEOREM 2.1. CCPV07, ADSY10 The correlation gap of a monotone submodular function is at most $e /(e-1)$.

\section{Posted-Price vs Optimal: A Reduction to Correlation Gap}

We will focus on comparing SPMs with the optimal mechanism in the context of revenue maximization. Almost identical claims can be made for welfare and certain other objectives, which we discuss in Section 3.3 .

\subsection{A Single Bidder Optimization Problem Be-} fore we embark on studying mechanisms that involve multiple bidders, it is crucial to first understand the following optimization problem that involves only one bidder.

Problem 3.1. Given an agent with valuation distribution $F$, and a target selling probability $0<q<1$, what price distribution $\mathcal{D}$ maximizes our expected revenue, i.e., $E_{p \sim \mathcal{D}}[p \cdot(1-F(p))]$, subject to the constraint that the selling probability is exactly $q$, i.e., $E_{p \sim \mathcal{D}}[1-F(p)]=q$.

To study this problem, first suppose that we can only offer a deterministic price. Then for any selling probability $q$, our only choice is to offer the deterministic

\footnotetext{
${ }^{1}$ We differ from ADSY10 in that the correlation gap was defined there to be at most 1 .
}

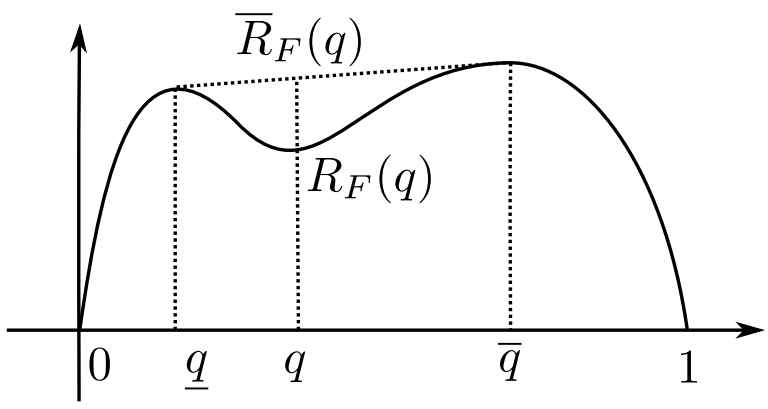

Figure 1: Revenue Curve and "Ironed" Revenue Curve

price $F^{-1}(1-q)$, and the revenue we get as a function of $q$ is $R_{F}(q)=q \cdot F^{-1}(1-q)$.

Now suppose instead we are allowed to offer a random price, then we can do possibly better. To be specific, we can randomize between two prices $p$ and $\bar{p}$ with selling probabilities $q=1-F(p)$ and $\bar{q}=\overline{1}-F(\bar{p})$ satisfying $\underline{q} \leq q \leq \bar{q}$, and in particular we draw $\underline{p}$ with probability $\frac{\bar{q}-q}{\bar{q}-\underline{q}}$ and draw $\bar{p}$ with probability $\frac{q-\underline{q}}{\bar{q}-\underline{q}}$ such that the selling probability is exactly equal to $q$. Then our revenue is equal to $\frac{\bar{q}-q}{\bar{q}-\underline{q}} \cdot R_{F}(\underline{q})+\frac{q-\underline{q}}{\bar{q}-\underline{q}} \cdot R_{F}(\bar{q})$, which is possibly better than $R_{F}(\bar{q})$. Let $\bar{R}_{F}(q) \overline{\text { be the maximum }}$ revenue one can get by randomizing between two prices this way. One can show that $\bar{R}_{F}$ equals to the concave closure of $R_{F}$, i.e., the minimum concave function that upper-bounds $R_{F}$. Moreover, the optimal distribution is in fact just the two-price distribution that gives $\bar{R}_{F}(q)$.

In the well-known special case that $F$ is regular, i.e., $R_{F}(q)$ is concave in $q^{2}$, the two-price distribution degenerates to a single deterministic price $F^{-1}(1-q)$, and $\bar{R}_{F}(q)=R_{F}(q)$ in this case.

For the purpose of the rest of the paper, the following lemma summarizes this discussion.

Lemma 3.1. [Mye81, BR89] For all valuation distribution $F$ and probability $q$, the price distribution $\mathcal{D}$ that maximizes $E_{p \sim \mathcal{D}}[p \cdot(1-F(p))]$ subject to the constraint that $E_{p \sim \mathcal{D}}[1-F(p)]=q$ is a two-price distribution, where this distribution as well as the revenue $\bar{R}_{F}(q)$ it gives us can be determined from $F$. Moreover, $\bar{R}_{F}(q)$ is a concave function.

For notational convenience, we will use $\bar{R}_{i}$ to denote the $\bar{R}_{F}$ function for agent $i$.

\subsection{Reduction Theorem: the Revenue Case}

Definition 3.1. The greedy-SPM of Chawla et al. [CHMS10] (with slight changes) does the following:

\footnotetext{
${ }^{2}$ This is equivalent to the definition that the virtual valuation function is monotone.
} 
1. For each agent $i$, calculate $q_{i}$, the winning probability of agent $i$ in Myerson's mechanism. Remove agent $i$ if $q_{i}=0$.

2. For each agent $i$, draw a random price $p_{i}$ from the optimal price distribution w.r.t. distribution $F_{i}$ and selling probability $q_{i}$ according to the Ironing Lemma.

3. Let $A=\emptyset$. For all agent $i$ in decreasing order of effective prices $\hat{p}_{i}$ defined as $\hat{p}_{i}=\bar{R}_{i}\left(q_{i}\right) / q_{i}$, if serving agent $i$ is feasible, i.e., $A+i \in \mathcal{I}$, offer price $p_{i}$ to agent $i$, and add $i$ into $A$ if agent $i$ accepts.

TheOrem 3.1. (Reduction Theorem For Matroids) For matroid environments, if the correlation gap of the weighted rank function is at most $\beta$ for no matter what non-negative weights, then the expected revenue greedy-SPM is a $\beta$-approximation to that of Myerson's optimal mechanism.

Proof. In the following two claims, we relate the expected revenue of both Myerson's mechanism and greedy-SPM to the weighted rank function with effective prices $\hat{p}_{i}$ as weights, which we denote as $\hat{p}^{*}$.

Claim 3.1. Let $W$ be the (random) set of winning agents in Myerson's mechanism. The expected revenue of Myerson's mechanism is upper-bounded by $E_{W}\left[\hat{p}^{*}(W)\right]$.

Proof. Let $q_{i}=\operatorname{Pr}_{W}[i \in W]$ be the probability that agent $i$ wins in Myerson's mechanism. By Lemma 3.1 the optimal way to sell to agent $i$ with probability $q_{i}$ gives expected revenue $\bar{R}_{i}\left(q_{i}\right)$. By linearity of expectation, the expected revenue of Myerson's mechanism is upper-bounded by $\sum_{i \in N} \bar{R}_{i}\left(q_{i}\right)$. To relate this to the effective prices, suppose in Myerson's mechanism, we get effective payment $\hat{p}_{i}$ whenever agent $i$ wins. Then the total effective revenue is $E_{W}\left[\sum_{i \in W} \hat{p}_{i}\right]$. Also, each agent $i$ wins with probability $q_{i}$ in Myerson's mechanism, contributing $q_{i} \hat{p}_{i}=\bar{R}_{i}\left(q_{i}\right)$ to total effective revenue, and hence $\sum_{i \in N} \bar{R}_{i}\left(q_{i}\right)$ equals effective revenue $E_{W}\left[\sum_{i \in W} \hat{p}_{i}\right]$. Further, since $W$ is a feasible set, we can rewrite $E_{W}\left[\sum_{i \in W} \hat{p}_{i}\right]$ as $E_{W}\left[\hat{p}^{*}(W)\right]$, and our claim follows.

Claim 3.2. Let demand set $D$ be the (random) set of agents whose values beat the prices set for them respectively. The expected revenue of greedy-SPM equals to $E_{D}\left[\hat{p}^{*}(D)\right]$.

Proof. Because valuation distributions of the agents are independent, each agent $i$ is in the demand set $D$ with probability $q_{i}$ independently. Observe that ignoring agents not in the demand set, who do not win anyway, greedy-SPM effectively runs the greedy algorithm on the demand set $D$ w.r.t. weights $\hat{p}_{i}$ subject to feasibility constraints. The expected effective revenue of greedySPM is hence equal to $E_{D}\left[\sum_{i \in \operatorname{greedy}(D)} \hat{p}_{i}\right]$, which is equal to $E_{D}\left[\hat{p}^{*}(D)\right]$ by the optimality of greedy for matroid. Note that whenever the random price $p_{i}$ is offered to an agent, we get expected revenue $\bar{R}_{i}\left(q_{i}\right)$, while the expected effective revenue is $q_{i} \hat{p}_{i}$, also equal to $\bar{R}_{i}\left(q_{i}\right)$. Therefore the expected revenue of greedySPM equals to the expected effective revenue, which is $E_{D}\left[\hat{p}^{*}(D)\right]$.

By our assumption that the correlation gap of the weighted rank function is at most $\beta$, we have $E_{D}\left[\hat{p}^{*}(D)\right] \geq \frac{1}{\beta} \cdot E_{W}\left[\hat{p}^{*}(W)\right]$, and our theorem follows by chaining this inequality with the above two claims.

For settings beyond matroids, we need the following technical condition for the reduction to work, which is a stronger condition than merely a bound on correlation gap.

DEFINITION 3.2. We say that the greedy algorithm verifies a correlation gap of $\beta$ for the weighted rank function of a set system, if for all nonnegative weights $\left(w_{i}\right)_{i \in N}$, and distribution $\mathcal{D}$ over $2^{N}$, we have $E_{S \sim \mathcal{I}(\mathcal{D})}\left[\sum_{i \in \operatorname{greedy}(S)} w_{i}\right] \geq \frac{1}{\beta} E_{S \sim \mathcal{D}}\left[w^{*}(S)\right]$.

TheOrem 3.2. (Reduction TheOrem In General) For any downward-closed environment, if the greedy algorithm verifies a correlation gap of $\beta$ for the weighted rank function for arbitrary non-negative weights, then the expected revenue of greedy-SPM is a $\beta$-approximation to that of Myerson's optimal mechanism.

Proof. Similarly, we upper-bound the revenue of Myerson by $E_{W}\left[\hat{p}^{*}(W)\right]$, and express the revenue of greedy$\mathrm{SPM}$ as $E_{D}\left[\sum_{i \in \operatorname{greedy}(D)} \hat{p}_{i}\right]$. The theorem follows by applying the assumption that greedy verifies a correlation gap of $\beta$.

REMARK 3.1. One crucial property about the greedy algorithm is that although we are running greedy on the all agents, but for no matter what demand set it turns out to be, greedy is also optimizing or approximately optimizing for this demand set. Most other approximation algorithms do not have this property.

3.3 Extension to Welfare and Other Objectives We specify an objective by defining functions of the form $g_{i}(v, p)$ for agents. If agent $i$ has true value $v$ and is offered a price $p$ with $v \geq p$, then agent $i$ wins, and 
we gain objective value $g_{i}(v, p)$. Our goal is then to maximize the total objective value we collect from the agents. For maximizing welfare, revenue, and surplus, we set $g_{i}(v, p)=v, g_{i}(v, p)=p$, and $g_{i}(v, p)=v-p$, respectively. One can also define other objectives this way.

To adapt the definition of greedy-SPM and our reduction theorems, we need the following changes. We define $G_{i}(q)$ as the maximum expected objective value the seller can get by offering a deterministic price such that the agent wins with probability $q$. We can then derive an Ironing Lemma similarly, and also define $\bar{G}_{i}(q)$ as the concave closure of $G_{i}(q)$. Then we use effective gain defined as $\bar{G}_{i}(q) / q$ to replace effective prices as weights, and the rest of the proof goes the same way.

3.4 Efficiently Computable SPMs In greedySPM, we need to compute the winning probabilities of the agents in Myerson's mechanism, which is potentially computationally hard. This was addressed in Chawla et al. by a sampling-based approach, which estimates the winning probabilities by repeatedly running Myerson's mechanism for sufficiently many times.

We note that the winning probabilities give a feasible solution to the following convex program, whose optimal value gives an upper bound on the revenue of Myerson's mechanism.

$$
\begin{array}{cc}
\operatorname{maximize} \sum_{i \in N} \bar{R}_{i}\left(q_{i}\right) & \\
\text { subject to } & \\
\sum_{i \in S} q_{i} \leq \operatorname{rank}(S) & \text { for all } S \\
q_{i} \geq 0 & \text { for all } i
\end{array}
$$

For many settings, we can solve this convex program efficiently to get the optimal $q_{i}$ values, and use them in greedy-SPM instead. It turns out that for settings we study in this paper, this variant of greedy-SPM gives the same approximation guarantees. We leave the details of this observation to the full version of the paper.

\section{Revenue and Welfare Guarantees of Greedy-SPM}

Based on the reduction theorem, we give tight analysis of greedy-SPM of Chawla et al., and prove the guarantees in Theorem 4.1. By the reduction theorem, it suffices to study the the correlation gaps of the weighted rank functions, and the greedy algorithm, which we do separately in the following subsections.

THEOREM 4.1. The expected revenue of greedy-SPM is a $\beta$-approximation to that of Myerson's optimal mechanism, and the expected welfare of (the welfare version of) greedy-SPM is a $\beta$-approximation to that of the $V C G$ mechanism, where:
- $\beta=e /(e-1)$ for matroid environments (an improvement over 2)

- $\beta=1 /\left(1-\frac{k^{k}}{e^{k} k !}\right) \approx 1 /\left(1-\frac{1}{\sqrt{2 \pi k}}\right)$ for $k$-unit auctions (an improvement over e/(e-1))

- $\beta=p+1$ for $p$-independent environments (a generalization from intersection of $p$ matroids)

REMARK 4.1. For matroid environments, as noticed in [CHMS10], if we run the VCG mechanism, and set reserves to be the same as the prices used in greedy$S P M$, the revenue we get is as good as that of greedy$S P M$, for any particular valuation profile. It follows that the VCG mechanism with such reserve prices has the same approximation guarantee for revenue.

4.1 Matroid Environments Matroid environments are important because many auction constraints can be modeled using matroids, and matroids have various nice properties. To give a few examples of matroids, $k$-uniform matroids encode the constraints of $k$-unit auctions, where $S$ is in $\mathcal{I}$ if and only if $|S| \leq k$, graphical matroids enforce the feasible sets to be the edge sets of acyclic subgraphs of a given graph, and transversal matroids can model certain matching markets, and etc.

By the reduction theorem, to establish an $e /(e-1)$ approximation of greedy-SPM in matroid environments, it suffices to prove the following lemma.

LEMMA 4.1. The correlation gap of the weighted rank function of a matroid is at most $e /(e-1)$.

Proof. This lemma follows from the fact that the weighted rank function of a matroid is monotone and submodular, and that the correlation gap of a monotone submodular function is at most $e /(e-1)$.

$4.2 k$-Unit Auctions $k$-Unit auctions form an important sub-class of a matroid environments. The feasibility constraints of a $k$-unit auction is modeled by a $k$-uniform matroid. In the following, we precisely quantify the correlation gap of the weighted rank function of $k$-uniform matroids.

For a $k$-uniform matroid over $n$ elements, the (unweighted) rank function is $f_{n}^{k}(S)=\min (|S|, k)$ for $S \subseteq N=\{1, \ldots, n\}$. We drop superscript and subscript when the context is clear. It is easy to verify that $f$ is monotone and submodular. Define the multi-linear extension $\operatorname{Ef}(\mathbf{q})$ for $\mathbf{q} \in[0,1]^{n}$ (in the sense of [CCPV07]) as the expectation of $f(S)$ where each $i \in N$ is included in $S$ with probability $q_{i}$ independently. As was shown in [CCPV07], or can be easily verified using definitions, if $f$ is submodular, then $E f$ satisfies cross-convexity, in the sense that $\frac{\partial^{2} E f(\mathbf{q})}{\partial q_{i} \partial q_{j}} \leq 0$ for all $\mathbf{q} \in(0,1)^{n}$ and $i \neq j$. 
For all $n$ and $0 \leq k \leq n$, define $\Phi(n, k)$ as the minimum of $E f_{n}^{k}(\mathbf{q})$ over all marginal probability vector $\mathbf{q}$ such that $\sum_{i \in N} q_{i}=k$. In the following lemma, we identify the probability vector $\mathbf{q}$ that minimizes $E f(\mathbf{q})$ subject to this constraint, and show several useful properties about $\Phi(n, k)$. This lemma is interesting in itself, and in fact can be used to improve the analysis of an SPM in SY10.

Lemma 4.2. The following holds for $\Phi(n, k)$ :

(a) $\Phi(n, k)=E f_{n}^{k}(\mathbf{q})$ where $q_{i}=k / n$ for all $i \in$ $\{1, \ldots, n\}$. In other words, $\Phi(n, k)$ is the expected value of $\min (X, k)$, where $X$ is a binomial random variable with parameters $n$ and $k / n$.

(b) $\Phi(n, k)$ monotonely increases with $k$, and monotonely decreases with $n$.

(c) $\lim _{n \rightarrow \infty} \Phi(n, k)=k-\frac{k^{k+1}}{e^{k} k !} \approx k-\frac{k}{\sqrt{2 \pi k}}$.

Proof. To prove (a), first for an arbitrary marginal probability vector $\mathbf{q} \in[0,1]^{n}$, consider vector $\overline{\mathbf{q}}$ that is the same as $\mathbf{q}$ except that the $i$-th and $j$-th components are averaged for some $i \neq j$, i.e., $\bar{q}_{i}=\bar{q}_{j}=\left(q_{i}+q_{j}\right) / 2$. We show that $E f(\overline{\mathbf{q}}) \leq E f(\mathbf{q})$. Let $\mathbf{q}^{\prime}$ be the same as q except with the $i$-th and $j$-th components switched, i.e., $q_{i}^{\prime}=q_{j}$ and $q_{j}^{\prime}=q_{i}$. By symmetry of $f, E f(\mathbf{q})=$ $E f\left(\mathbf{q}^{\prime}\right)$, and $\overline{\mathbf{q}}$ is the middle-point of $\mathbf{q}$ and $\mathbf{q}^{\prime}$. By the cross-convexity of $E f$, the value of $E f$ is convex in the line segment connecting $\mathbf{q}$ and $\mathbf{q}^{\prime}$. Therefore $E f(\overline{\mathbf{q}})$ is at most the average of $E f(\mathbf{q})$ and $E f\left(\mathbf{q}^{\prime}\right)$, or simply $E f(\mathbf{q})$. Now starting with an arbitrary $\mathbf{q}$, by repeatedly averaging the maximum and minimum components of $\mathbf{q}$ this way, the value of $E f(\mathbf{q})$ keeps decreasing, while all $q_{i}$ 's converge to $k / n$. By the continuity of $E f(\mathbf{q})$ in $\mathbf{q}$, the value of $E f(\mathbf{q})$ converges to the value of $E f$ at $q_{i}=k / n$ for all $i$. Therefore $E f(\mathbf{q})$ is minimized at $q_{i}=k / n$ for all $i$.

To show (b), it is obvious that $\Phi(n, k)$ is monotonely increasing in $k$, because $f_{n}^{k}(S)$ is increasing in $k$. It suffices to show that $\Phi(n, k)$ is monotonely decreasing in $n$. Recall that $\Phi(n, k)$ was defined to be the optimal value of a minimization problem. To relate $\Phi(n, k)$ to $\Phi(n+1, k)$, we cast the optimal solution underlying $\Phi(n, k)$, which is an $n$-dimensional independent distribution, to $(n+1)$-dimensional space, such that it gives a candidate solution to the minimization problem underlying $\Phi(n+1, k)$. To be specific, we observe that $\Phi(n, k)$ is equal to $E f_{n+1}^{k}(\mathbf{q})$, where $\mathbf{q}$ is an $(n+1)$-dimensional vector with $q_{i}=k / n$ for $i=1, \ldots, n$, and $q_{n+1}=0$. By definition of $\Phi(n+1, k)$, $\Phi(n+1, k) \geq E f_{n+1}^{k}(\mathbf{q})=\Phi(n, k)$.

We leave the derivation of (c) to the appendix.
Based on Lemma 4.2, we can first quantify the correlation gap of the unweighted rank function, and then extend it to the weighted case.

Lemma 4.3. For $n, k \geq 1$, the correlation gap of the function $f(S)=\min (|S|, k)$ for $S \subseteq N=\{1, \ldots, n\}$ is exactly $\frac{k}{\Phi(k, n)}$.

Proof. For any probability vector $\mathbf{q}$, let $\mathcal{O}_{\mathbf{q}}$ be the distribution over $2^{N}$ with marginal probabilities $\mathbf{q}$ that maximizes $E_{S \sim \mathcal{O}_{\mathbf{q}}}[f(S)]$. We first show that $E_{S \sim \mathcal{O}_{\mathbf{q}}}[f(S)]$ equals $\sum_{i} q_{i}$ if $\sum_{i} q_{i} \leq k$, and equals $k$ otherwise. (1) Suppose $\sum_{i} q_{i} \leq k$. First note that $E_{S \sim \mathcal{O}_{\mathbf{q}}}[f(S)] \leq$ $E_{S \sim \mathcal{O}_{\mathbf{q}}}[|S|]=\sum_{i} q_{i}$. Moreover, $\mathbf{q}$ can be seen as a point inside the integral polytope with (characteristic vectors of) feasible sets (sets of size at most $k$ ) as vertices. Then by standard polyhedral combinatorics, one can decompose this point as a convex combination of the vertices, which corresponds to a distribution over feasible sets with marginal probabilities q. This distribution gives expected $f$ value $\sum_{i} q_{i}$. (2) If $\sum_{i} q_{i}>k$, then by the monotonicity of $E_{S \sim \mathcal{O}_{\mathbf{q}}}[f(S)]$ in $\mathbf{q}, E_{S \sim \mathcal{O}_{\mathbf{q}}}[f(S)]$ is at least $k$. However it is also upper-bounded by $k$ as $f$ is upper-bounded by $k$. Therefore $E_{S \sim \mathcal{O}_{\mathrm{q}}}[f(S)]=k$ in this case.

Suppose that q maximizes the "gap ratio" $\frac{E_{S \sim \mathcal{O}_{\mathrm{q}}}[f(S)]}{E_{S \sim \mathrm{q}}[f(S)]}$. We first show that $r=\sum_{i} q_{i} \leq k$. If this is not the case, then by lowering the $q_{i}$ 's such that $\sum_{i} q_{i}=k, E_{S \sim \mathbf{q}}[f(S)]$ strictly decreases, while $E_{S \sim \mathcal{O}_{\mathrm{q}}}[f(S)]$ is still $k$. This gives a strictly higher gap ratio, contrary to that assumption that $q$ maximizes the gap ratio.

Next we show that $r=k$. For $r \leq k$, we can explicitly express the reciprocal of the gap ratio as:

$$
\begin{aligned}
& \frac{1}{r} \cdot \sum_{t=0}^{n}\left(\begin{array}{c}
n \\
t
\end{array}\right) \cdot\left(\frac{r}{n}\right)^{t} \cdot\left(\frac{n-r}{n}\right)^{n-t} \cdot \min (t, k) \\
= & \sum_{t=1}^{n}\left(\begin{array}{c}
n-1 \\
t-1
\end{array}\right)\left(\frac{r}{n}\right)^{t-1}\left(\frac{n-r}{n}\right)^{n-t} \cdot \frac{\min (t, k)}{t}
\end{aligned}
$$

This is equal to the expectation of $\frac{\min (X+1, k)}{X+1}$ where $X$ is the binomial random variable with parameters $n-1$ and $r / n$. It is also equal to $\int_{0}^{\infty} \operatorname{Pr}\left[\frac{\min (X+1, k)}{X+1} \geq x\right] d x$. Note that for $x>1, \operatorname{Pr}\left[\frac{\min (X+1, k)}{X+1} \geq x\right]=0$, and otherwise $\operatorname{Pr}\left[\frac{\min (X+1, k)}{X+1} \geq x\right]=\operatorname{Pr}[X+1 \leq k / x]$, where $\operatorname{Pr}[X+1 \leq k / x]$ strictly decreases as $r$ increases. Therefore the gap ratio is maximized at $r=k$.

Lemma 4.4. For $n, k \geq 1$, the correlation gap of the weighted rank function of a $k$-uniform matroid of size $n$ is at most $\frac{k}{\Phi(k, n)}$. 
Proof. Again let $f(S)=\min (|S|, k)$ for $S \subseteq N=$ $\{1, \ldots, n\}$. Assume w.l.o.g. that $w_{1} \geq w_{2} \geq \ldots \geq w_{n}$, and let $w_{n+1}=0$ for convenience. The weighted rank function $w^{*}(S)$ can be written as $\sum_{i \in N}\left(w_{i}-w_{i+1}\right)$. $f(S \cap\{1, \ldots, i\})$, a conic combination of unweighted rank functions. The correlation gap of $w^{*}$ is therefore witnessed by the correlation gap of $f(S \cap\{1, \ldots, i\})$ for some $i$, and hence it equals $\sup _{1 \leq i \leq n} k / \Phi(i, k)$. By Lemma 4.2(b), $\Phi(i, k)$ is decreasing in $i$, and hence the correlation gap of $w^{*}$ is $k / \Phi(n, k)$.

REMARK 4.2. We cannot generalize Lemma 4.3 or 4.4 to work for arbitrary matroids with rank $k$. For any $k$, consider the partition matroids with $k$ parts, each of size $n$, where a feasible set can only have at most one element from each part. The rank of such a matroid is $k$, while the correlation gap is the same as that of a 1-uniform matroid over $n$ elements, which approaches $e /(e-1)$ as $n$ increases.

$4.3 p$-Independent Environments There are interesting auction constraints that cannot be modeled by matroids, but can be modeled by $p$-independent set systems. In a set system $(N, \mathcal{I})$, a base of a subset $S \subseteq N$ is a maximal feasible subset of $S$. A set system $(N, \mathcal{I})$ is a $p$-independent system if for any non-empty subset $S$ of $N$ :

$$
\frac{\text { maximum size of a base of } S}{\text { minimum size of a base of } S} \leq p \text {. }
$$

For example, a matroid is 1-independent, and vice versa. The edge sets of (non-bipartite) matchings of a graph form a 2-independent system (but in general cannot be cast as the intersection of a constant number of matroids). The intersection of $p$ matroids is $p$ independent. The feasible sets of agents in singleminded combinatorial auctions with bounded bundle size $p$ form a $p$-independent system.

It is well-known that the greedy algorithm gives a $p$-approximation for $p$-independent systems [Jen76]. For our purpose, it suffices to prove the following lemma, by combining arguments of [CCPV07, CHMS10].

LEMma 4.5. The greedy algorithm verifies a correlation gap of $p+1$ for $p$-independent system constraints.

This ratio of $p+1$ is tight, up to lower order terms.

Proposition 4.1. For any sufficiently large positive integer $p$, there is a p-independent set system with correlation gap at least $p / \log p$.

\section{Conclusion}

We summarize the main observation of this paper as follows. For revenue and welfare maximization, the approximation ratio of certain SPM compared to the optimal mechanism is inherently related to the correlation gap of the weighted rank function of set system that models the feasibility constraints. In particular for matroid environments, the weighted rank functions have small correlation gap, which explains why SPMs give good approximation guarantees in these settings.

Moreover, our point is made stronger by the fact that we are proving guarantees for a very restricted type of SPMs, where prices and offering order have to be predetermined. Our observation can be used as a guideline for the design and analysis of more relaxed types of SPMs, which seems to be an interesting research direction.

Acknowledgment This research is made possible by learning from two "right" groups of people, people behind SPMs: Shuchi Chawla, Jason Hartline, David Malec, and Balu Sivan, and people behind correlation gap: Shipra Agrawal and Jan Vondrák. I thank them for sharing their intuition about the subject with me. I also thank Tim Roughgarden, who knows both subjects, for various valuable suggestions.

\section{References}

[ADSY10] S. Agrawal, Y. Ding, A. Saberi, and Y. Ye. Correlation robust stochastic optimization. In Proc. 21th ACM Symp. on Discrete Algorithms, 2010.

[AM06] L. M. Ausubel and P. Milgrom. The lovely but lonely vickrey auction. In Combinatorial Auctions, chapter 1. MIT Press, 2006.

[BBDS11] Moshe Babaioff, Liad Blumrosen, Shaddin Dughmi, and Yaron Singer. Posting prices with unknown distributions. In Innovations in Computer Science (ICS), 2011.

[BH08] L. Blumrosen and T. Holenstein. Posted prices vs. negotiations: an asymptotic analysis. In Proc. 9th ACM Conf. on Electronic Commerce (EC), 2008.

[BR89] J. Bulow and J. Roberts. The simple economics of optimal auctions. The Journal of Political Economy, 97:1060-90, 1989.

[CCPV07] G. Calinescu, C. Chekuri, M. Pál, and J. Vondrák. Maximizing a submodular set function subject to a matroid constraint. In SIAM Journal on Computing, special issue for STOC 2008, 2007.

$\left[\mathrm{CEDG}^{+} 10\right]$ T. Chakraborty, E. Even-Dar, S. Guha, Y. Mansour, and S. Muthukrishnan. Approximation schemes for sequential posted pricing in multi-unit auctions. In Workshop on Internet and Network Economics (WINE), 2010.

[CHMS10] S. Chawla, J. Hartline, D. Malec, and B. Sivan. Sequential posted pricing and multi-parameter mechanism design. In Proc. 39th ACM Symp. on Theory of Computing (STOC), 2010. 
[Cla71] E. H. Clarke. Multipart pricing of public goods. Public Choice, 11:17-33, 1971.

[DRS09] S. Dughmi, T. Roughgarden, and M. Sundararajan. Revenue submodularity. In Proc. 10th ACM Conf. on Electronic Commerce (EC), pages 243-252, 2009.

[DRY10] P. Dhangwatnotai, T. Roughgarden, and Q. Yan. Revenue maximization with a single sample. In Proc. 11th ACM Conf. on Electronic Commerce (EC), pages 129-138, 2010.

[Gro73] T. Groves. Incentives in teams. Econometrica, 41:617-631, 1973.

[Hol08] C. Holahan. Auctions on eBay: A dying breed, 2008.

[HR09] J. D. Hartline and T. Roughgarden. Simple versus optimal mechanisms. In Proc. 10th ACM Conf. on Electronic Commerce (EC), pages 225-234, 2009.

[Jen76] T.A. Jenkyns. The efficiency of the greedy algorithm. In Proceedings of the 7th South Eastern Conference on Combinatorics, Graph Theory and Computing, pages 341-350. ACM, 1976.

[Mye81] R. Myerson. Optimal auction design. Mathematics of Operations Research, 6(1):58-73, 1981.

[NR00] N. Nisan and A. Ronen. Computationally feasible vcg mechanisms. In Proc. 2nd ACM Conf. on Electronic Commerce (EC), pages 242-252. ACM Press, 2000.

[Ox192] J. G. Oxley. Matroid Theory. Oxford, 1992.

[SY10] M. Sundararajan and Q. Yan. Robust mechanisms for risk-averse sellers. In Proc. 11th ACM Conf. on Electronic Commerce (EC), pages 139-148, 2010.

[Vic61] W. Vickrey. Counterspeculation, auctions, and competitive sealed tenders. J. of Finance, 16:8-37, 1961.

[Von07] J. Vondrák. Submodularity in combinatorial optimization. Phd thesis, Charles University, Prague, 2007.

[Von08] J. Vondrák. Optimal approximation for the submodular welfare problem in the value oracle model. In Proc. 39th ACM Symp. on Theory of Computing (STOC), pages 67-74, 2008.

\section{Proof of Lemma 4.2}

Proof. We derive the asymptotics for $\Phi(n, k)$ as follows, where the last step is by Stirling's approximation of factorials.

$$
\begin{aligned}
& \lim _{n \rightarrow \infty} \Phi(n, k) \\
= & \lim _{n \rightarrow \infty} \sum_{t=0}^{n}\left(\begin{array}{l}
n \\
t
\end{array}\right) \cdot\left(\frac{k}{n}\right)^{t} \cdot\left(\frac{n-k}{n}\right)^{n-t} \cdot \min (t, k) \\
= & \lim _{n \rightarrow \infty} \sum_{t=0}^{k-1}\left(\begin{array}{l}
n \\
t
\end{array}\right) \cdot\left(\frac{k}{n}\right)^{t} \cdot\left(\frac{n-k}{n}\right)^{n-t} \cdot t \\
& +k \cdot\left(1-\sum_{t=0}^{k-1}\left(\begin{array}{l}
n \\
t
\end{array}\right) \cdot\left(\frac{k}{n}\right)^{t} \cdot\left(\frac{n-k}{n}\right)^{n-t}\right)
\end{aligned}
$$

$$
\begin{aligned}
& =\sum_{t=0}^{k-1} \frac{k^{t}}{t !} \cdot \frac{1}{e^{k}} \cdot t+k \cdot\left(1-\sum_{t=0}^{k-1} \frac{k^{t}}{t !} \cdot \frac{1}{e^{k}}\right) \\
& =k \cdot\left(1-\frac{k^{k}}{e^{k} k !}\right) \approx k \cdot\left(1-\frac{1}{\sqrt{2 \pi k}}\right) .
\end{aligned}
$$

\subsection{Proof of Lemma 4.5}

Proof. Fix marginal probabilities q. In the dependent case, if $S$ is drawn from a distribution $\mathcal{D}$ with marginal probabilities $\mathbf{q}$, let $\tilde{q}_{i}$ be the probability that $i$ is in the optimal feasible subset of $S$ (with arbitrary fixed tiebreaking). we can rewrite $E_{S \sim \mathcal{D}}\left[w^{*}(S)\right]$ as $\sum_{i \in N} \tilde{q}_{i} w_{i}$.

Now consider the independent case, where each $i$ is in $S$ with probability $q_{i}$ independently, which we denote by $S \sim$ q. Let $A=g(S)$ be the agents allocated by running the greedy algorithm on $S$. The expected performance of greedy is $E_{S \sim \mathcal{D}}\left[\sum_{i \in A} w_{i}\right]$. An equivalent way of looking at running the greedy algorithm on the random set $S$ is the following:

1. $A=\emptyset$

2. visit all agents $i \in N$ in decreasing order of weights:

(a) if $A+i \in \mathcal{I}$, we check if $i$ is in $S$, and add $i$ into $A$ if yes.

(b) if $A+i \notin \mathcal{I}$, we ignore $i$.

3. output $A$

Let random set $U$ be the set of agents that are ignored by greedy. Consider the quantity $Q=E_{S \sim \mathbf{q}}\left[\sum_{i \in A} w_{i}+\right.$ $\left.\sum_{i \in U} \tilde{q}_{i} w_{i}\right]$. For every agent $i$, if she is checked by greedy, she contributes $q_{i} w_{i}$ to the $Q$. (with probability $q_{i}, i$ is in $S$, and we get weight $w_{i}$ ) On the other hand, if she is ignored, she contributes $\tilde{q}_{i} w_{i}$ to $Q$. Therefore,

$Q=E_{S \sim \mathbf{q}}\left[\sum_{i \in A} w_{i}+\sum_{i \in U} \tilde{q}_{i} w_{i}\right] \geq \sum_{i \in N} \tilde{q}_{i} w_{i}=E_{S \sim \mathcal{D}}\left[w^{*}(S)\right]$.

Next we show that $w(A) \geq \frac{1}{p} \sum_{i \in U} \tilde{q}_{i} w_{i}$, and our theorem would follow as:

$$
\begin{aligned}
E_{S \sim \mathbf{q}}\left[\sum_{i \in \operatorname{greed} y(S)} w_{i}\right] & =E_{S \sim \mathbf{q}}\left[\sum_{i \in A} w_{i}\right] \\
& \geq \frac{1}{p+1} E_{S \sim \mathcal{D}}\left[w^{*}(S)\right] .
\end{aligned}
$$

Let $A$ contain $i_{1}, i_{2}, \ldots, i_{l}$ in the order of inclusion into $A$ by greedy. Partition $U$ into $B_{j}$ 's for $j=1, \ldots, l$, where $B_{j}$ is the set of agents ignored by greedy after $i_{1}, \ldots, i_{j}$ have been added into $A$. Therefore $w_{i} \leq w_{i_{j}}$ for $i \in B_{j}$. Consider the set $\left\{i_{1}, \ldots, i_{j}\right\} \cup B_{1} \cup \ldots \cup B_{j}$. At any time step, greedy's solution set is always a maximal feasible subset of the agents visited so far. 
Therefore $\left\{i_{1}, \ldots, i_{j}\right\}$ is a base of $\left\{i_{1}, \ldots, i_{j}\right\} \cup B_{1} \cup \ldots \cup$ $B_{j}$. By the definition of $p$-independence, the maximal base of $\left\{i_{1}, \ldots, i_{j}\right\} \cup B_{1} \cup \ldots \cup B_{j}$ has size at most $p \cdot j$, and it follows that $\sum_{i \in B_{1} \cup \ldots \cup B_{j}} \tilde{q}_{i} \leq p \cdot j$.

Now our claim $\sum_{i \in A} w_{i} \geq \frac{1}{p} \sum_{i \in U} \tilde{q}_{i} w_{i}$ follows from the following inequalities: (let $w_{i_{l+1}}=0$ )

$$
\begin{aligned}
\sum_{i \in U} \tilde{q}_{i} w_{i} & =\sum_{1 \leq j \leq l} \sum_{i \in B_{j}} \tilde{q}_{i} w_{i} \\
& \leq \sum_{1 \leq j \leq l} \sum_{i \in B_{j}} \tilde{q}_{i} w_{i_{j}} \\
& =\sum_{1 \leq j \leq l} \sum_{i \in B_{1} \cup \ldots \cup B_{j}} \tilde{q}_{i}\left(w_{i_{j}}-w_{i_{j+1}}\right) \\
& \leq \sum_{1 \leq j \leq l} p \cdot j \cdot\left(w_{i_{j}}-w_{i_{j+1}}\right) \\
& =p \cdot \sum_{1 \leq j \leq l} w_{i_{j}}=p \cdot \sum_{i \in A} w_{i} .
\end{aligned}
$$

\subsection{Proof of Proposition 4.1}

Proof. To define the set system $(N, \mathcal{I})$, let $Y$ be the set of all strings $a_{1} a_{2} \ldots a_{n}$ of length $n$ over the alphabet $\{1, \ldots, n\}$. For every $i \in\{1,2, \ldots, n\}$ and $b \in\{1, \ldots, n\}$, we denote by $\left[a_{i}=b\right]$ the "miniset" that contains all strings from $Y$ with the $i$-th letter $a_{i}$ being $b$. Then $N$ is the set of all such minisets. To define the feasible subsets $\mathcal{I}$, a subset $S$ of minsets from $N$ is feasible if and only if no two minisets in $S$ intersect. Note that two different minisets $\left[a_{i}=b\right]$ and $\left[a_{i^{\prime}}=b^{\prime}\right]$ intersect if and only if $i \neq i^{\prime}$. It is easy to verify that this set system is $n$-independent. Finally, we assign unit weights to every miniset.

We choose a random subset $S$ of $N$ in two ways. In the dependent case, an index $i$ from $\{1, \ldots, n\}$ is chosen at random, and $S$ contains the miniset $\left[a_{i}=b\right]$ for all $b \in\{1, \ldots, n\}$. Clearly all such $S$ 's are feasible, and the rank function has expected value $n$.

In the independent case, for all $i, b$, we include every miniset $\left[a_{i}=b\right]$ in $S$ with probability $1 / n$ independently. For all $i$, let $X_{i}$ be the number of minisets in $S$ that have the form $\left[a_{i}=b\right]$ for some $b$. Then the rank function is equal to $\max _{i} X_{i}$. To give a rough estimate of $E\left[\max _{i} X_{i}\right]$, note that for all $i$,

$$
\begin{aligned}
& \operatorname{Pr}\left[X_{i} \geq \frac{1}{2} \log n\right] \\
= & \sum_{k=\frac{1}{2} \log n}^{n}\left(\begin{array}{l}
n \\
k
\end{array}\right)\left(\frac{1}{n}\right)^{k}\left(1-\frac{1}{n}\right)^{n-k} \\
\leq & \sum_{k=\frac{1}{2} \log n}^{n}\left(\frac{n \cdot e}{k}\right)^{k} \frac{1}{n^{k}} \leq n \cdot\left(\frac{e}{\frac{1}{2} \log n}\right)^{\frac{1}{2} \log n}
\end{aligned}
$$

$$
=\frac{n}{2^{\Omega(\log n \cdot \log \log n)}} .
$$

Therefore for sufficiently large $n, \operatorname{Pr}\left[\max _{i} X_{i} \geq\right.$ $\left.\frac{1}{2} \log n\right] \leq 1-\left(1-\frac{n}{2^{\Omega(\log n \cdot \log \log n)}}\right)^{n} \leq \frac{1}{n}$, and hence $E\left[\max _{i} X_{i}\right] \leq \operatorname{Pr}\left[\max _{i} X_{i} \geq \frac{1}{2} \log n\right] \cdot n+\frac{1}{2} \log n \leq \log n$. It follows that the correlation gap is at least $n / \log n$ for sufficiently large $n$. 